\title{
Effects of Different Controlling Factors on The Experimental Landform Development
}

Shunji Ouchi

Chuo University, Tokyo, Japan

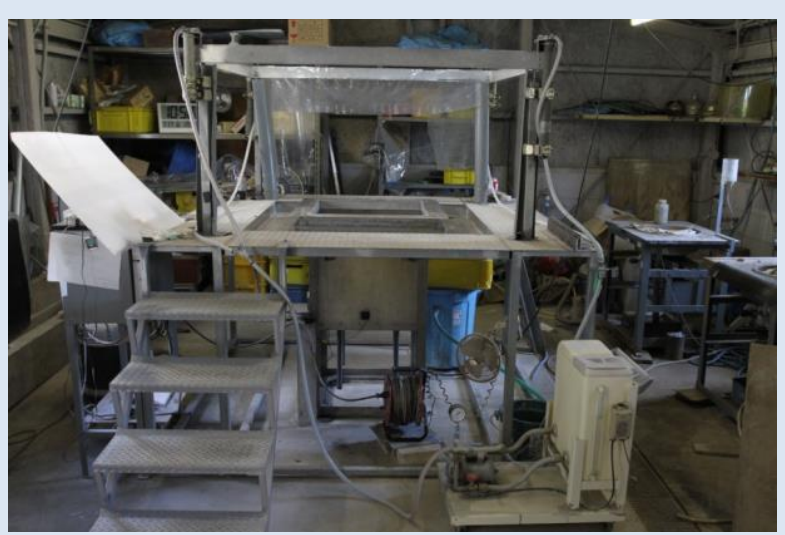

\section{Experimental facilities}
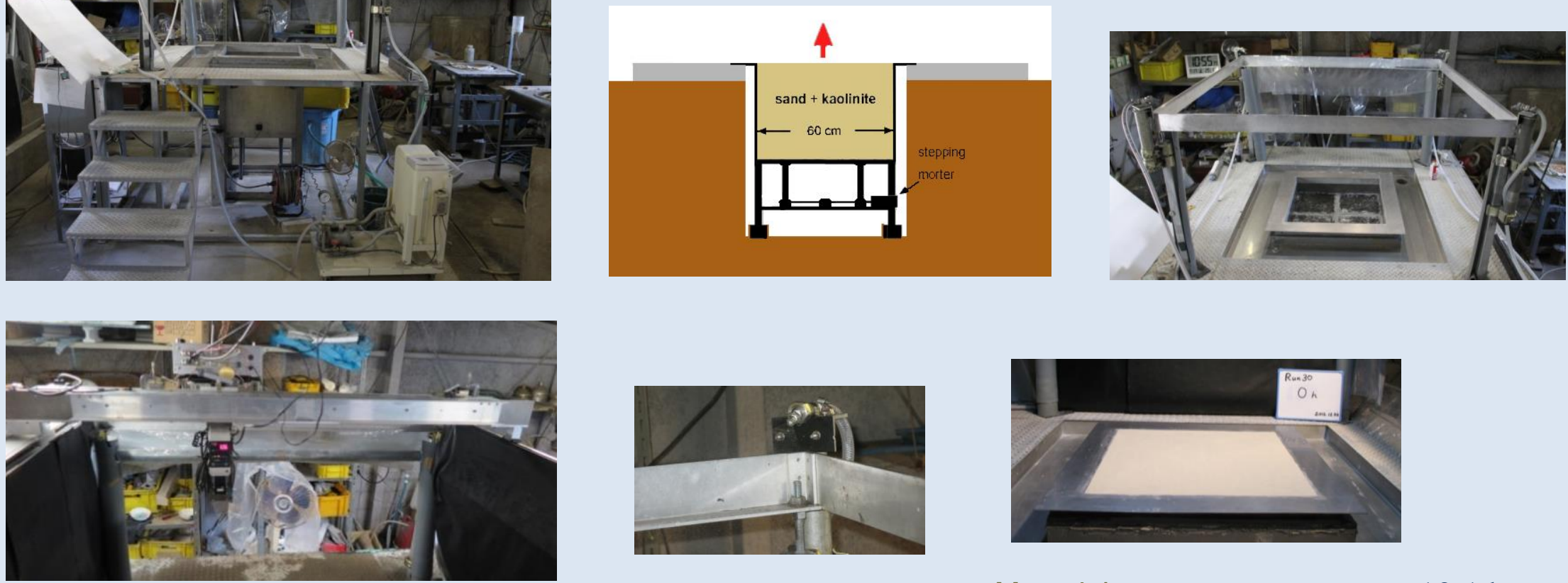

Material : fine sand : kaolinite $=10: 1$ by weight 
Duration of

uplift/rainfall

hours

Average rainfall

Uplift rate

Total

Permeability

Width of

$\mathrm{mm} / \mathrm{h}$

uplift

$\mathrm{cm} / \mathrm{s}$

deposition area

\begin{tabular}{|lllllll|}
\hline Run26 & $1000 / 1000$ & $40-50$ & $0.36 \mathrm{~mm} / \mathrm{h}$ & $358 \mathrm{~mm}$ & $3.2 \times 10^{-4}$ & $100 \mathrm{~mm}$ \\
\hline Run27 & $970 / 1000$ & $80-90$ & $0.36 \mathrm{~mm} / \mathrm{h}$ & $348 \mathrm{~mm}$ & $2.9 \times 10^{-4}$ & $100 \mathrm{~mm}$ \\
\hline Run28 & $176 / 256$ & $80-90$ & $2.0 \mathrm{~mm} / \mathrm{h}$ & $354 \mathrm{~mm}$ & $3.5 \times 10^{-4}$ & $100 \mathrm{~mm}$ \\
\hline Run29 & $72 / 160$ & $80-90$ & $5.0 \mathrm{~mm} / \mathrm{h}$ & $361 \mathrm{~mm}$ & $2.8 \times 10^{-4}$ & $100 \mathrm{~mm}$ \\
\hline Run30 & $1160 / 1160$ & $80-90$ & $0.1 \mathrm{~mm} / \mathrm{h}$ & $118 \mathrm{~mm}$ & $3.0 \times 10^{-4}$ & $100 \mathrm{~mm}$ \\
\hline Run31 & $1160 / 1160$ & $80-90$ & $0.1 \mathrm{~mm} / \mathrm{h}$ & $117 \mathrm{~mm}$ & $4.7 \times 10^{-4}$ & $200 \mathrm{~mm}$ \\
\hline Run32 & $1000 / 1000$ & $80-90$ & $0.36 \mathrm{~mm} / \mathrm{h}$ & $364 \mathrm{~mm}$ & $1.8 \times 10^{-4}$ & $200 \mathrm{~mm}$ \\
\hline Run33 & $120 / 176$ & $80-90$ & $3.0 \mathrm{~mm} / \mathrm{h}$ & $363 \mathrm{~mm}$ & $2.9 \times 10^{-4}$ & $200 \mathrm{~mm}$ \\
\hline Run34 & $184 / 184$ & $80-90$ & $2.0 \mathrm{~mm} / \mathrm{h}$ & $368 \mathrm{~mm}$ & $4.2 \times 10^{-4}$ & $200 \mathrm{~mm}$ \\
\hline Run35 & $1000 / 1168$ & $80-90$ & $0.36 \mathrm{~mm} / \mathrm{h}$ & $364 \mathrm{~mm}$ & $4.7 \times 10^{-4}$ & $50,100,200 \mathrm{~mm}$ \\
\hline Run36 & $176 / 432$ & $80-90$ & $2.0 \mathrm{~mm} / \mathrm{h}$ & $354 \mathrm{~mm}$ & $2.1 \times 10^{-4}$ & $200 \mathrm{~mm}$ \\
\hline Run37 & $176 / 336$ & $80-90$ & $2.0 \mathrm{~mm} / \mathrm{h}$ & $355 \mathrm{~mm}$ & $2.0 \times 10^{-3}$ & $200 \mathrm{~mm}$ \\
\hline Run38 & $960 / 1540$ & $80-90$ & $0.36 \mathrm{~mm} / \mathrm{h}$ & $348 \mathrm{~mm}$ & $1.5 \times 10^{-3}$ & $200 \mathrm{~mm}$ \\
\hline Run39 & $960 / 1540$ & $40-50$ & $0.36 \mathrm{~mm} / \mathrm{h}$ & $349 \mathrm{~mm}$ & $1.4 \times 10^{-3}$ & $200 \mathrm{~mm}$ \\
\hline Run40 & $960 / 1540$ & $80-90$ & $0.36 \mathrm{~mm} / \mathrm{h}$ & $348 \mathrm{~mm}$ & $3.0 \times 10^{-4}$ & $200 \mathrm{~mm}$ \\
\hline
\end{tabular}


Controlling factors considered: Uplift - uplift rate, Erosion - rainfall intensity,

- width of deposition area,

- permeability

- shear strength

\section{Uplift rate}
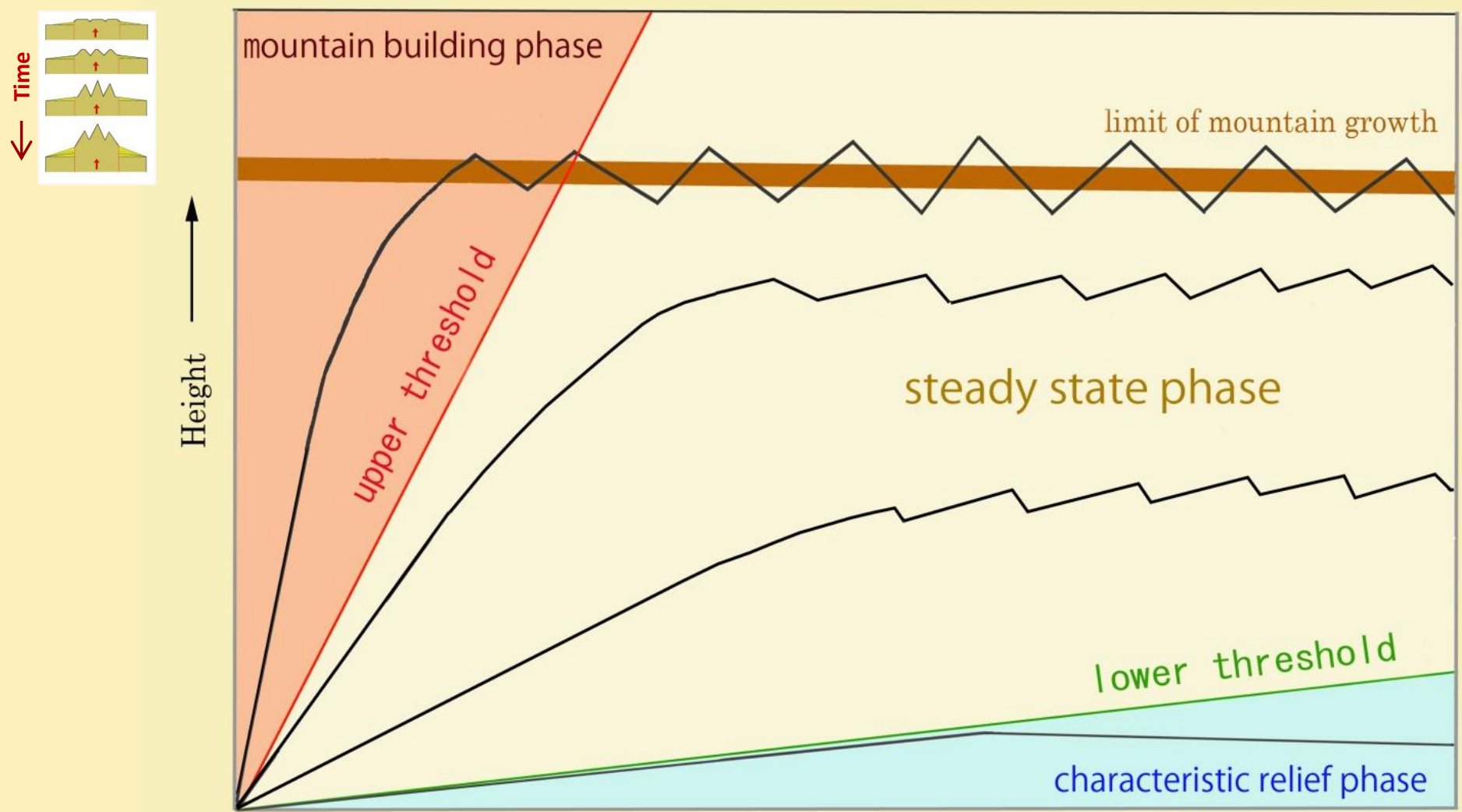

$\underbrace{+i}_{i=1}$

Time 
Uplift rate $=5.0 \mathrm{~mm} / \mathrm{h}$

\section{Mountain building phase}

Run 27

Jplift rate $=0.36 \mathrm{~mm} / \mathrm{h}$

Steady state phase

Uplift rate $=0.1 \mathrm{~mm} / \mathrm{h}$

Characteristic relief phase

(nearly)

Run 30 

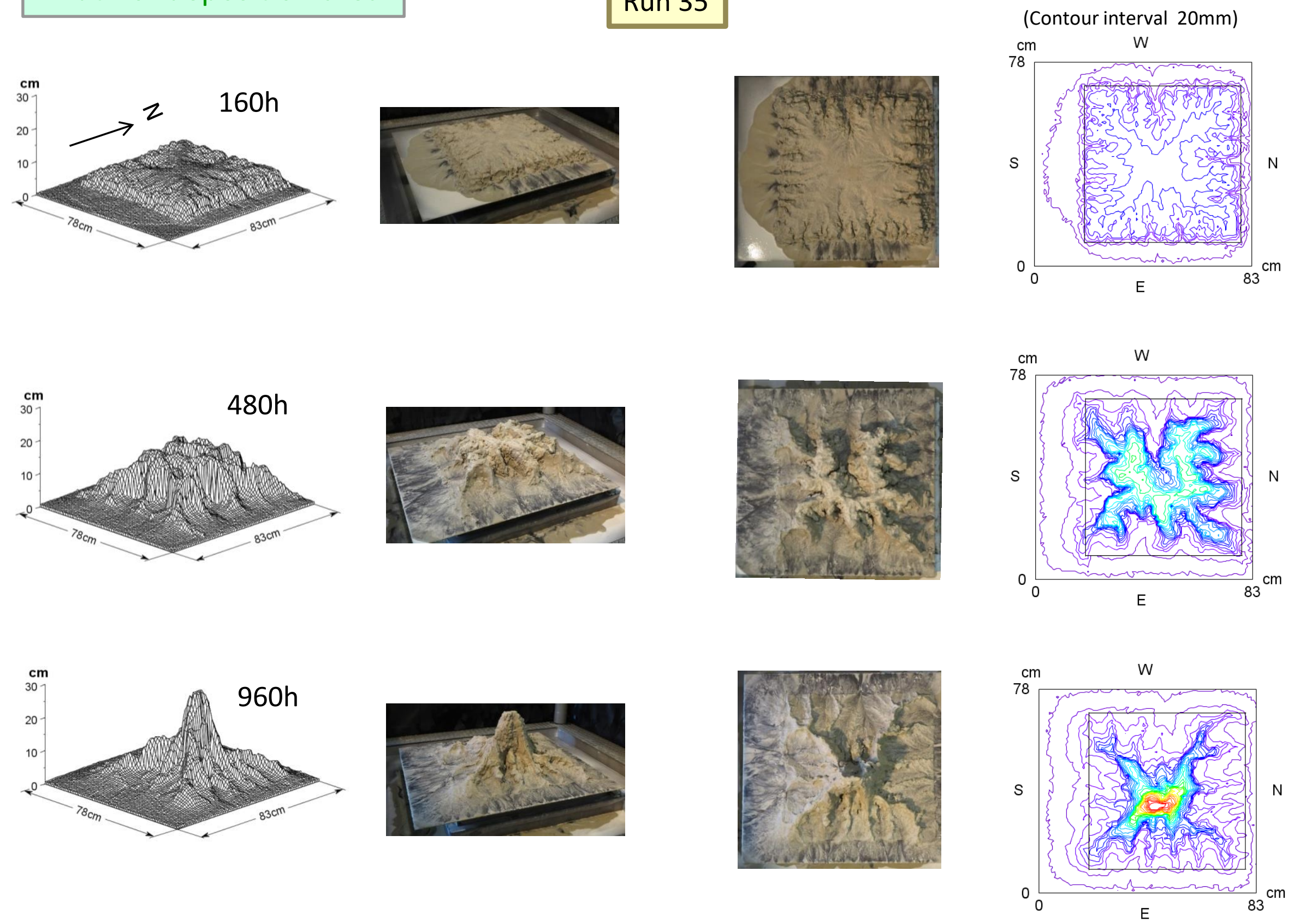


\section{Rainfall intensity}

precipitation permeability

deposition area

Run26 40-50 mm/h $\mathbf{3 . 2} \times \mathbf{1 0}^{-4} \mathrm{~cm} / \mathrm{s} 100 \mathrm{~mm}$

Run27 80-90 mm/h $2.9 \times \mathbf{1 0}^{-4} \mathrm{~cm} / \mathrm{s} \quad 100 \mathrm{~mm}$

Run 26 120h
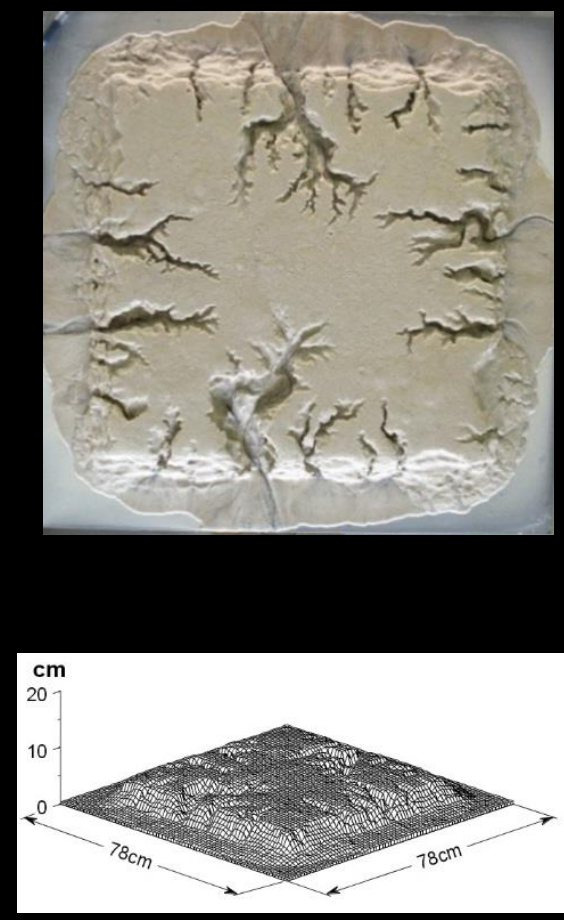

Run 27 120h
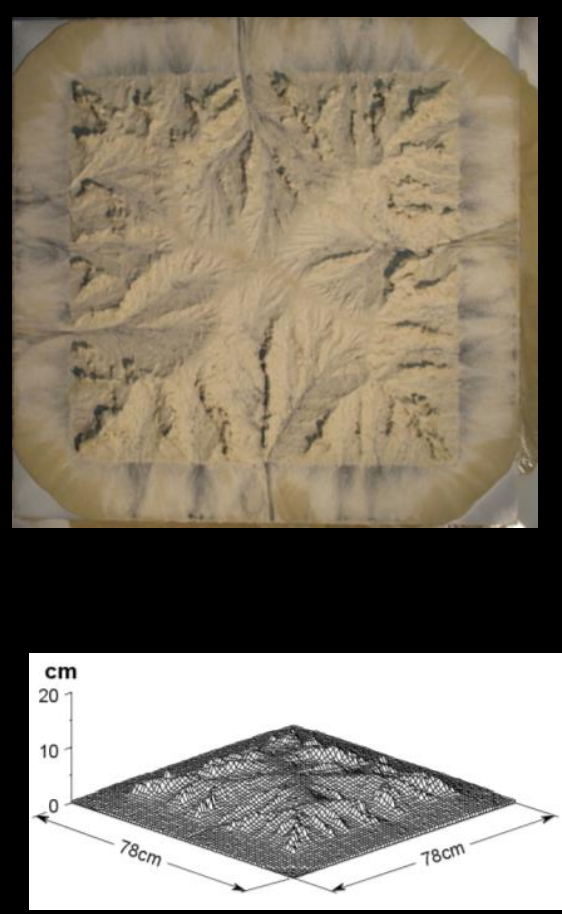

Change in average height (zmean)

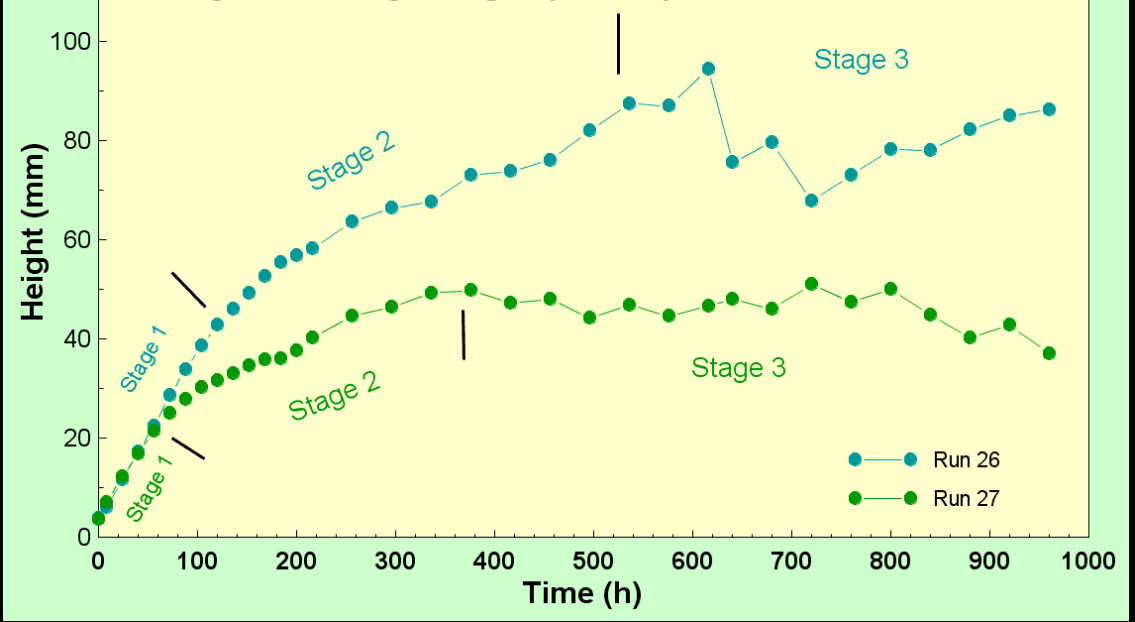

Run 26 640h
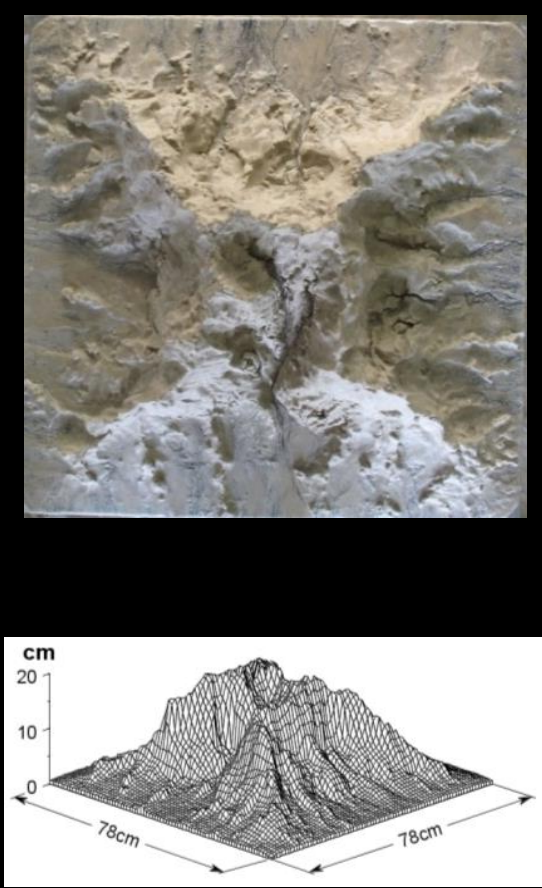

Run 27 640h
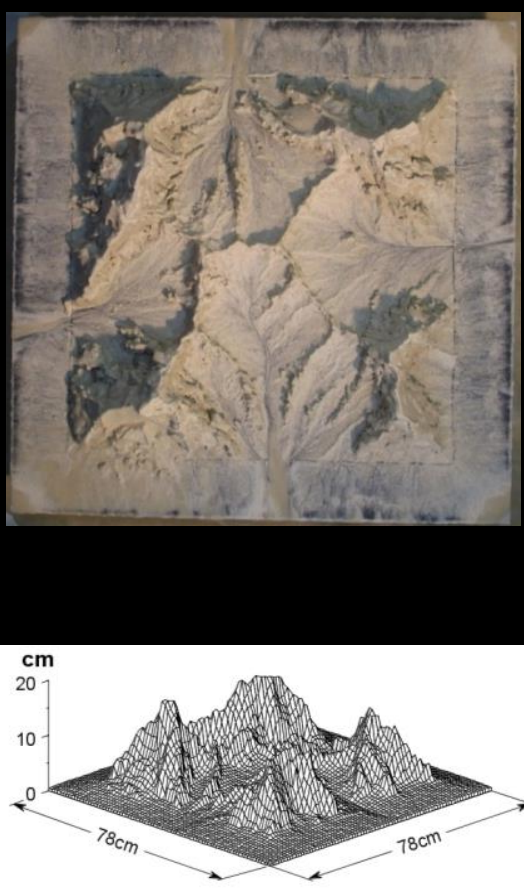


\section{Rainfall intensity}

Permeability and shear strength

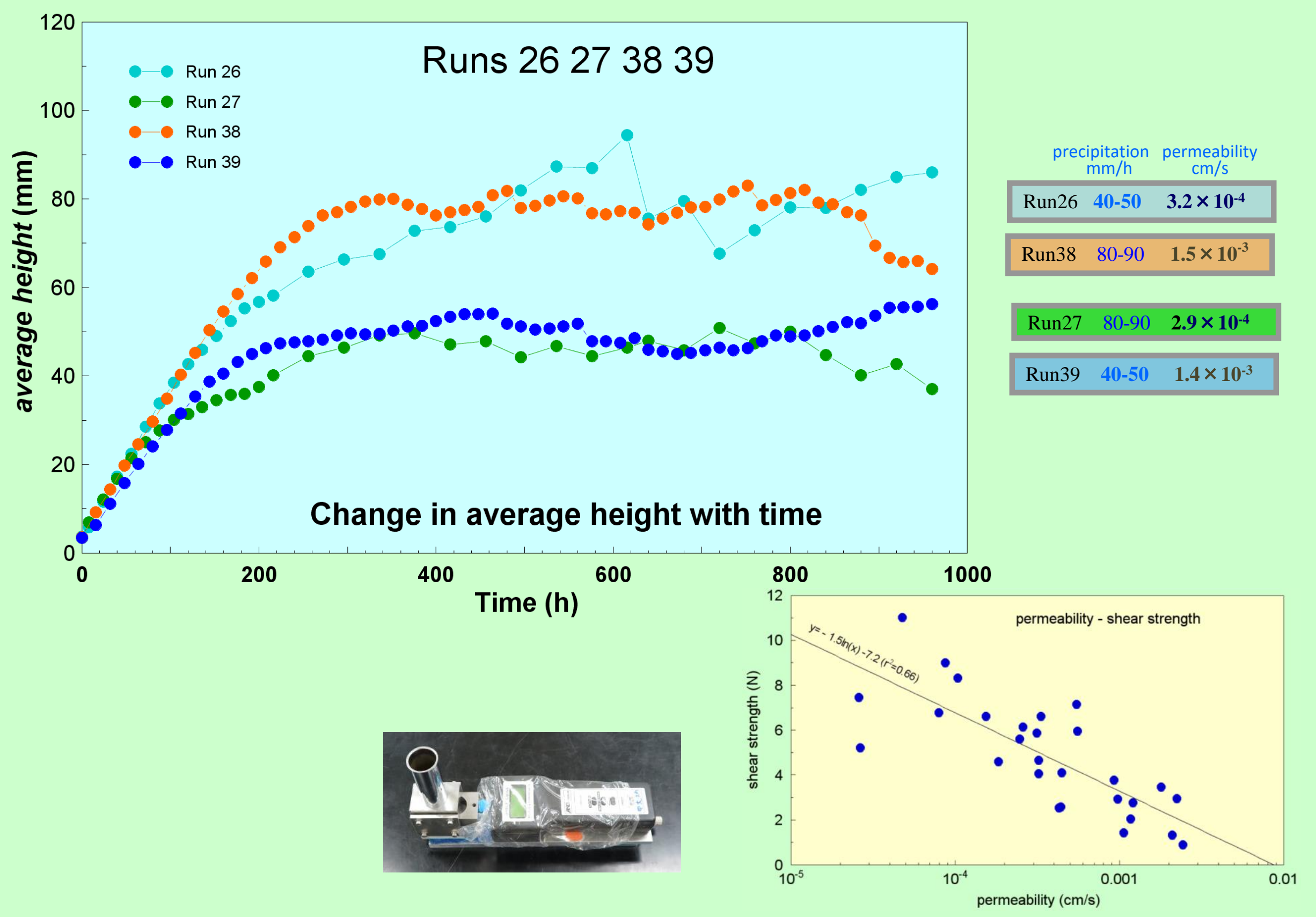


*water available for possible Hortonian overland flow

(precipitation - permeability)

\begin{tabular}{|l|}
\hline Lower rainfall \\
\hline \hline Lower permeability \\
\hline
\end{tabular}
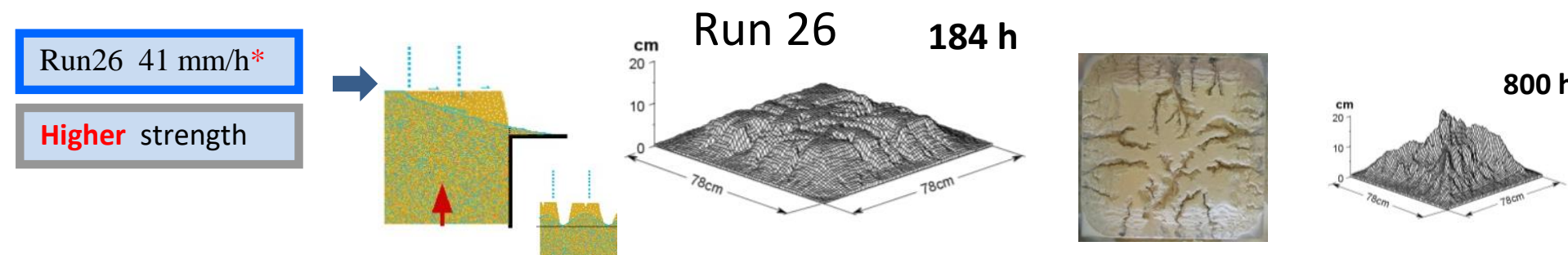

\begin{tabular}{|l|}
\hline Higher rainfall \\
\hline Higher permeability \\
\hline
\end{tabular}
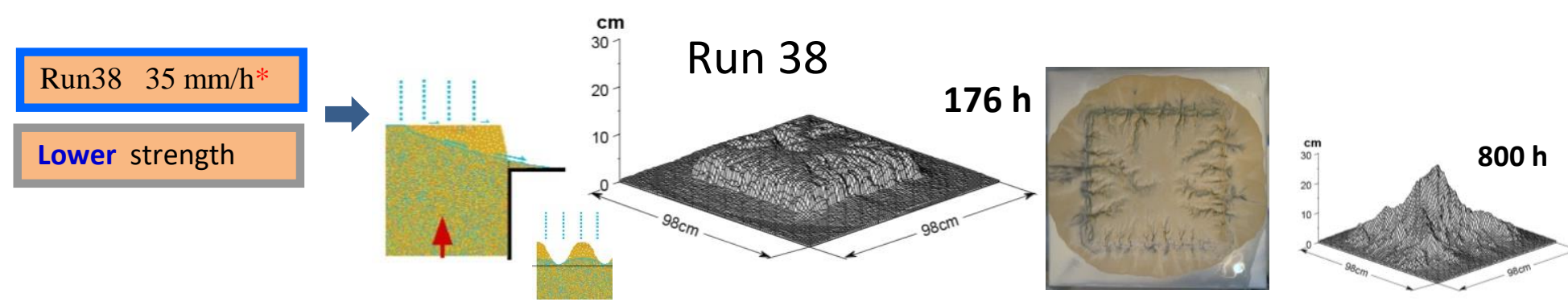

\section{Higher rainfall}

\section{Lower permeability}

Lower rainfall

Higher permeability
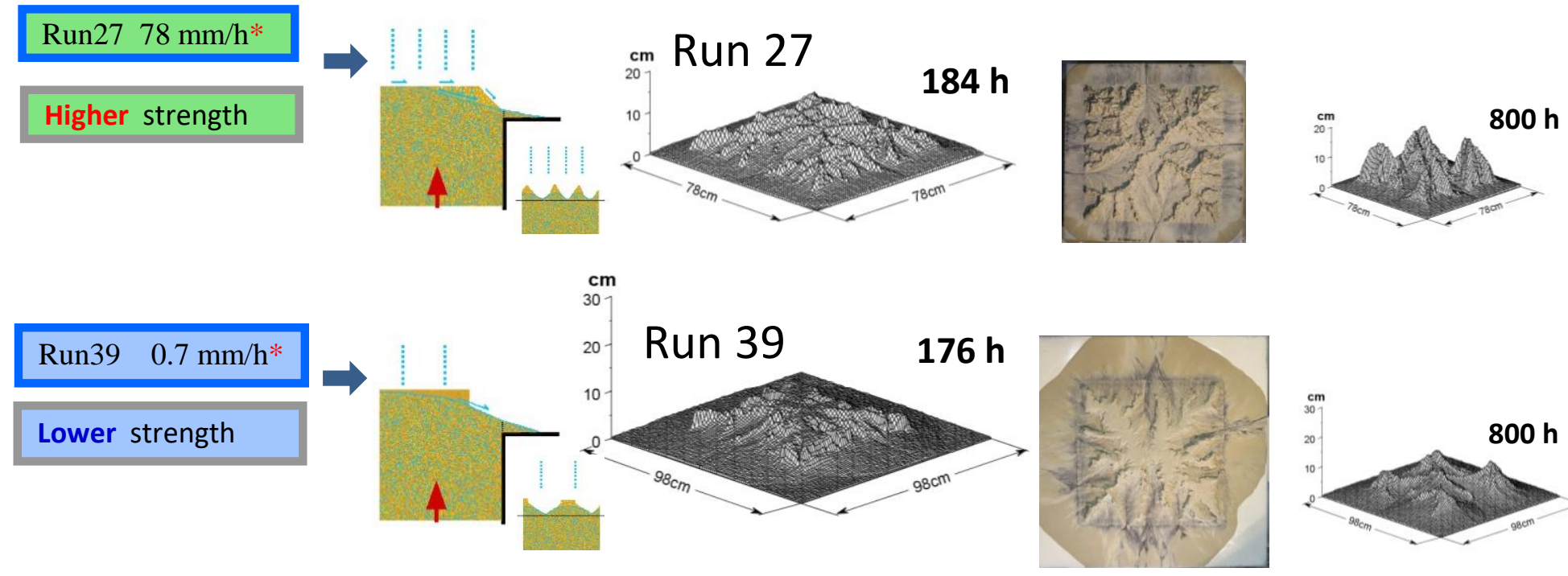


\section{Percentage hypsometric curves}
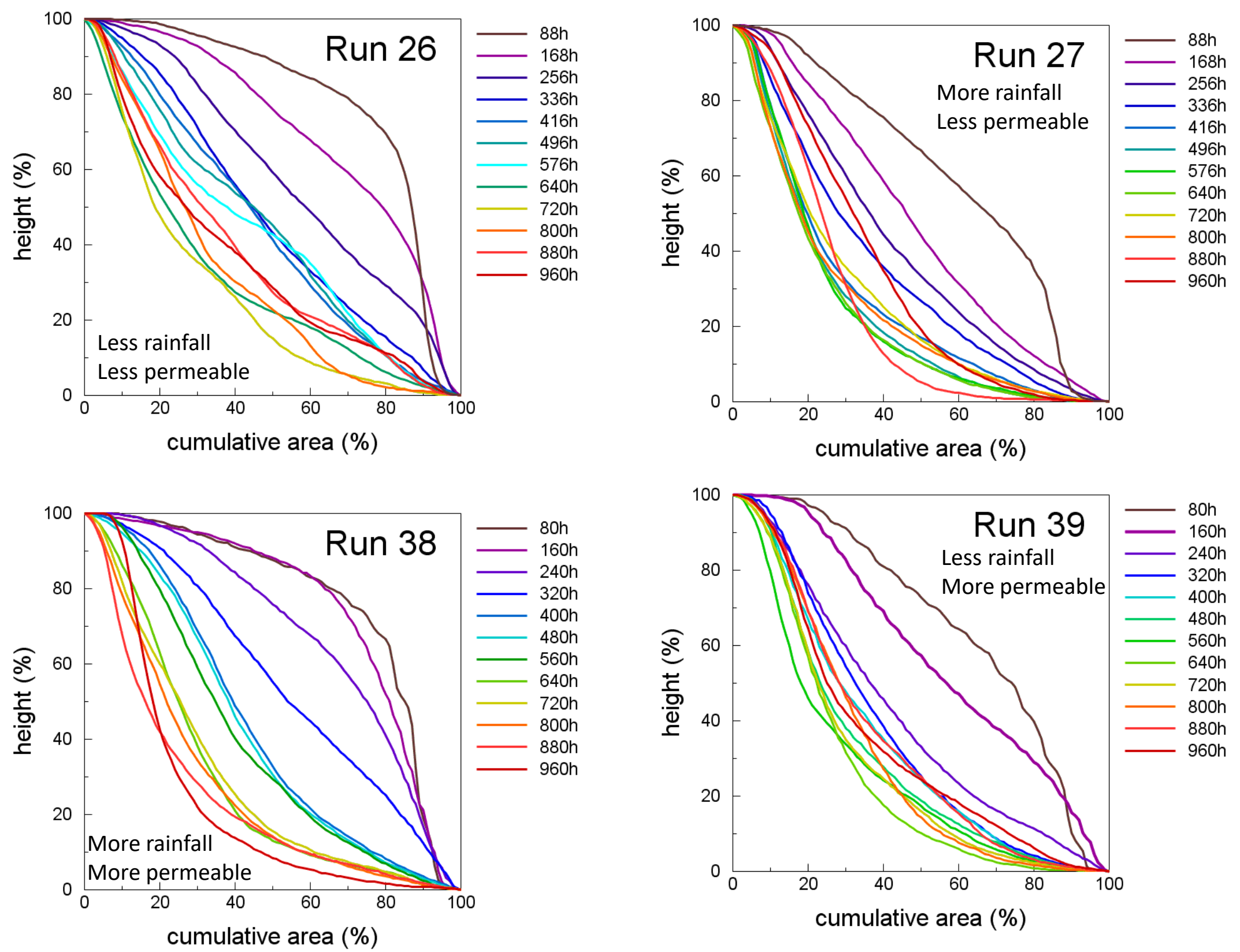


\section{Concluding remarks}

- Factors, such as uplift rate, rainfall intensity, permeability and shear strength, have certain significant effects on the way of experimental landform development. Their effects, however, appear in a complicated way with possible interactions among these controlling factors.

- Landform development is a complicated process even in the simplified experimental setting. Real landforms that develop in geographical space through geological/historical time with the almost infinite number of controlling factors, therefore, seems to be hopelessly complicated. 